\title{
A Mobile Computing Method Using CNN and SR for Signature Authentication with Contour Damage and Light Distortion
}

\author{
Mei Wang $\mathbb{D},{ }^{1}$ Ke Zhai, ${ }^{1}$ Chi Harold Liu, ${ }^{2,3}$ and Yujie Li $\mathbb{D}{ }^{4}$ \\ ${ }^{1}$ School of Electrical and Control Engineering, Xian University of Science and Technology, Xian, China \\ ${ }^{2}$ School of Software, Beijing Institute of Technology, China \\ ${ }^{3}$ Department of Computer Information and Security, Sejong University, Republic of Korea \\ ${ }^{4}$ School of Information Engineering, Yangzhou University, Yangzhou 225127, China \\ Correspondence should be addressed to Mei Wang; wangm@xust.edu.cn and Yujie Li; yzyjli@gmail.com
}

Received 21 November 2017; Accepted 21 March 2018; Published 25 June 2018

Academic Editor: Haider Abbas

Copyright (c) 2018 Mei Wang et al. This is an open access article distributed under the Creative Commons Attribution License, which permits unrestricted use, distribution, and reproduction in any medium, provided the original work is properly cited.

\begin{abstract}
A signature is a useful human feature in our society, and determining the genuineness of a signature is very important. A signature image is typically analyzed for its genuineness classification; however, increasing classification accuracy while decreasing computation time is difficult. Many factors affect image quality and the genuineness classification, such as contour damage and light distortion or the classification algorithm. To this end, we propose a mobile computing method of signature image authentication (SIA) with improved recognition accuracy and reduced computation time. We demonstrate theoretically and experimentally that the proposed golden global-local (G-L) algorithm has the best filtering result compared with the methods of mean filtering, medium filtering, and Gaussian filtering. The developed minimum probability threshold (MPT) algorithm produces the best segmentation result with minimum error compared with methods of maximum entropy and iterative segmentation. In addition, the designed convolutional neural network $(\mathrm{CNN})$ solves the light distortion problem for detailed frame feature extraction of a signature image. Finally, the proposed SIA algorithm achieves the best signature authentication accuracy compared with CNN and sparse representation, and computation times are competitive. Thus, the proposed SIA algorithm can be easily implemented in a mobile phone.
\end{abstract}

\section{Introduction}

Artificial intelligence influences the information technology being developed in today's world. People use artificial intelligence digital information technology almost anywhere and at any time. This supports daily social life and economic activities and contributes greatly to the sustainable growth of the economy and solves various social problems [1]. A signature is a commonly used human feature for identity authentication [2-4]. Artificial intelligence approaches to signature authentication have been evolving from offline methods to online methods to meet modern demands.

Regarding offline methods, researchers have developed signature recognition methods using a fusion algorithm involving distance and centroid orientation [5]. Additionally, the single optimized dehazing method has been proposed to estimate atmospheric light and remove the haze from an image [6], and methods to ensure consistency of signature verification have been proposed [7-10]. Dissimilarity normalization, shape features, and complex network spectrums have also been developed to assist in signature verification [11-14]. In addition, scientists presented a multitask metric learning recognition method that uses true and fake samples to calculate the similarity and dissimilarity of a signature [15]. Fine geometric structures were encoded by using a mesh template and splitting the area of the subset of features to analyze and verify a signature $[16,17]$.

Regarding online methods, scientists have utilized fast Fourier transform (FFT) [18] and dynamic time warping (DTW) [19] methods for on-air signature verification based on video, leading to the discrete cosine transform developed for online signature verification [20]. Researchers have also developed a signature alignment verification method to obtain a best match effect on the basis of a Gaussian mixture 


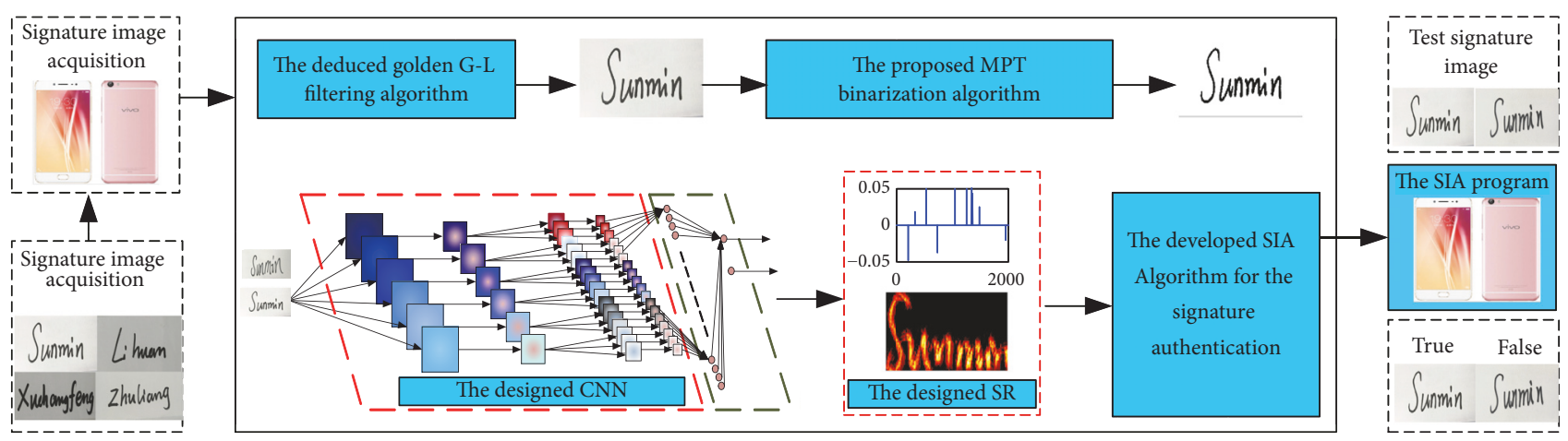

FIGURE 1: Signature authentication scheme.

algorithm [21]. Scientists have further developed various image-processing approaches, such as image descattering, color restoration, and image quality assessments that support signature verification activities.

Current state-of-the-art methods include deep learning and artificial intelligence. Recently, the convolutional neural network $(\mathrm{CNN})$ method has become a popular research topic in many applications. Additionally, researchers have proposed deep probabilistic neural networks and optimal parameters determined by particle swarm optimization (PSO) as a signature method design. In more modern applications, the traditional template signature has been replaced by the hidden signature to minimize the mean misalignment. Scientists have applied CNN to establish a fast level set algorithm to solve the color distortion problem for wound image intensity correction and segmentation processing, which could have applications in signature verification [22].

Deep CNN was originally developed for object classification, and reinforcement learning was developed to detect abnormal information [23]. For signature image authentication (SIA), scientists have proposed the improved CNN method to process distorted samples and decrease the distortion $[24,25]$. Sparse representation (SR) has been proposed for a separate feature-level fusion to integrate multiple feature representation [26]. Also, the local SR was proposed to improve robustness in cases of partial occlusion, deformation, and rotation in visual tracking [27]. The hierarchical SR was developed for synthetic aperture radar image classification [28]. In addition, researchers have developed the probabilistic class structure [29] and the sparse exponent batch processing method [30] combined with artificial intelligence methods for signature verification.

Meanwhile, mobile systems have been developing rapidly, and the above methods have not considered signature authentication applications for mobile systems, especially mobile phones. Based on the lack of such research, this paper addresses the problems of contour damage and light distortion as well as the classification accuracy of signature images. A combination of $\mathrm{CNN}$ and $\mathrm{SR}$ is proposed as a potential signature authentication method for mobile phones.

The organization of this paper is as follows: Section 2 presents the basic design for the signature authentication system, the golden global-local (G-L) filtering algorithm to solve the contour damage problem, the minimum probability threshold (MPT) segmentation algorithm to obtain the minimum error result, the $\mathrm{CNN}$ design for decreasing the light distortion, and the SIA algorithm to increase the recognition accuracy and obtain better speed performance. Section 3 discusses the experiments and comparisons. Finally, Section 4 concludes the paper.

\section{Materials And Methods}

The proposed system collects signature image samples using a mobile phone with the CamScanner app. Then ACDSee software is used for image cutting creating an image size of $64 \times 128$ pixels. Furthermore, MATLAB is used to conduct the CNN training and the SR method design. Finally, the SIA result is obtained and presented as output.

We design the signature authentication system scheme as shown in Figure 1. First, true signature images are collected by the mobile phone. Then, the signature verification system is applied on the basis of the deduced golden G-L filtering algorithm, the proposed MPT algorithm, and the proposed SIA algorithm. Finally, the application program of the SIA is installed in a mobile phone.

\subsection{Derivation Of Golden G-L Filtering Algorithm For Contour} Damage Mending. In order to mend the contour damage, remove noise, and smooth the signature image, a filtering process is needed first. Filtering is a convolution process of the input signature image with a core. Commonly used filtering methods are Gaussian filtering and mean filtering.

However, the Gaussian filtering effect should be improved, while mean filtering lacks scaling properties in variance and rotation symmetry. Therefore, we developed the golden G-L filtering algorithm below.

An original signature image, $I_{0}=f(m \times n)$, occupies the total area $S_{0}=(m \times n)$. The global mean gray value $M_{0}$ of image $I_{0}$ is

$$
M_{0}=\frac{1}{m \times n} \sum_{(i, j) \in S_{0}} f(i, j)
$$

where $f$ is the gray value of a pixel and $(i, j)$ are the coordinates of a pixel, and $m$ and $n$ are the row number 
TABLE 1: Deduced golden G-L filtering algorithm for mending contour damage of signature image.

\begin{tabular}{ll}
\hline Input: & The original signature image $I_{0}=f(x, y)$. \\
\hline Output: & The filtered image $I_{1}(x, y)$. \\
\hline Step 1: & $\begin{array}{l}\text { For each pixel of } I_{0}, \text { calculate the global mean gray value } M_{0} \text { and the variance } \delta^{2} \\
\text { according to }(1) \text { and }(2), \text { respectively. }\end{array}$ \\
\hline Step 2: & Calculate the local mean gray value $M_{1}$ of the pixel according to (3). \\
\hline Step 3: & Calculate the G-L mean gray value $M_{\mathrm{g}}$ of the pixel according to (4). \\
\hline Step 4: & Calculate the improved Gaussian template according to (5) and (6). \\
\hline Step 5: & Add salt and pepper noise signature image $I_{0}$ and obtain the filtered image $I_{1}$. \\
\hline Step 6: & $\begin{array}{l}\text { For each pixel, filter the noise signature image } I_{1} \text { by the convolution operation using } \\
\text { the improved Gaussian template } G(x, y), \text { and obtain the filtered image } I_{2} .\end{array}$ \\
\hline
\end{tabular}

and the column number of the original signature image $I_{0}$, respectively.

The gray value variance $\delta^{2}$ of the original signature image $I_{0}=f(m \times n)$ is

$$
\delta^{2}=\frac{1}{m \times n-1} \sum_{k=1}^{m \times n}\left(M-M_{0}\right)^{2}
$$

where $M_{0}$ is the global mean gray value of image $I_{0}, M_{1}$ is the local mean gray value of a pixel, and $m$ and $n$ were defined previously.

In order to obtain a better filtering effect, we define the G$\mathrm{L}$ mean parameter $M_{\mathrm{g}}$ by a combination of the mean filtering method with a golden section number $\sigma=0.618$.

The local neighbor area $S_{1}$ of a pixel is selected to be five rows and five columns, so it occupies the local area $S_{0}=(5 \times$ $5)$. The local mean gray value $M_{1}$ of image $I_{0}$ is then

$$
M_{1}=\frac{1}{5 \times 5} \sum_{(i, j) \in S_{1}} f(p, q)
$$

where the pixel coordinates are $(p, q)$ and $f$ is the gray value of a pixel.

According to experiments we conducted, a new parameter $M_{\mathrm{g}}$ of the G-L mean gray value is defined below.

$$
M_{\mathrm{g}}(x, y)=(1-\sigma) M_{0}+\sigma M_{1}
$$

where $x=1,2, \ldots, m, y=1,2, \ldots, n, \sigma$ is the golden section number $(\sigma=0.618), S_{0}$ and $S_{1}$ are the global area and the local area of a pixel, respectively, and $(x, y),(i, j)$, and $(p, q)$ represent pixel coordinates.

The G-L mean $M_{\mathrm{g}}$ is defined as the weighted sum of the global mean gray value $M_{0}$ and the local mean gray value $M_{1}$ with the golden section $\sigma$, and the local mean gray value $M_{1}$ is the main component of the G-L mean $M_{\mathrm{g}}$.

Thus, we determine the pixel positions $\left\{\left(i_{0}, j_{0}\right), \ldots,\left(i_{k}, j_{k}\right), \ldots\right\}$ where the gray values are equal to $M_{\mathrm{g}}$.

$$
\begin{aligned}
& \underset{\left(i_{k}, j_{k}\right)}{\operatorname{argmin}} \text { distance }\left[(x, y),\left(i_{k}, j_{k}\right)\right] \\
& \quad:=\left\{\left(i_{k}, j_{k}\right) \mid \forall\left(i_{k}, j_{k}\right): M\left(i_{k}, j_{k}\right)=M_{\mathrm{g}}\right\}
\end{aligned}
$$

where $(x, y)$ are the coordinates of a pixel of the original signature image $I_{0}, M$ is the gray value of a pixel, $M_{0}$ is the global mean gray value of image $I_{0}$, and $\left(i_{k}, j_{k}\right)$ are the pixel coordinates where the gray value is equal to $M_{\mathrm{g}}$.

Then, we design the improved Gaussian template $G(x, y)$ as below.

$$
\begin{array}{r}
G(x, y)=\frac{1}{4 \pi \delta^{2}} \exp \left(-\frac{\left(x-i_{k}\right)^{2}+\left(y-j_{k}\right)^{2}}{4 \delta^{2}}\right), \\
x=1, \ldots, m, y=1, \ldots, n
\end{array}
$$

Finally, the G-L filtering algorithm can be described on the basis of the defined parameter $M_{\mathrm{g}}$ and the improved Gaussian template $G(x, y)$ as outlined in Table 1 .

Note that salt and pepper noise is added to the original signature image $I_{0}$ in Step 5. This operation is used for mending any contour damage in the signature.

The deduced golden G-L filtering algorithm has scaling advantages in variance and rotation symmetry as well as the contour damage mending effect. This is because of the combination of the mean filtering, the Gaussian filtering and golden section of the global and local information, and the mending operation using salt and pepper noise, respectively.

\subsection{Proposed MPT Algorithm For Minimum Error Segmen-} tation. To achieve the minimum error binary segmentation of a signature image, we propose the MPT algorithm on the basis of signature image analysis and the developed optimal threshold for binary segmentation.

Image segmentation sets all pixel values to 0 or 1 while the pixel positions remain invariant. This operation simplifies postprocessing. Using this technique, the binary threshold influences the result dramatically. For example, Figure 2 shows the gray signature image and the histogram as well as the influence of a threshold on the segmentation results.

For a signature image, the gray degree histogram has two peaks. One is for the background of the signature, and the other is for the signature itself. Between these two peaks, there must be a minimum point where the gray degree value $M_{\text {min }}$ corresponds to the minimum histogram value. We select this minimum gray degree value $M_{\min }$ to be the threshold for binary segmentation of the filtered image $I_{2}(x, y)$. To ensure the minimum error segmentation of a signature image, the proposed MPT algorithm is described in Table 2. 
TABLE 2: Proposed MPT segmentation algorithm with minimum error.

\begin{tabular}{ll}
\hline Input: & The filtered image $I_{2}(x, y)$. \\
\hline Output: & The binarized image $I_{3}(x, y)$. \\
\hline Step 1: & $\begin{array}{l}\text { Calculate the gray degree distribution of the filtered signature image } I_{2}(x, y), \text { and } \\
\text { draw the histogram. }\end{array}$ \\
\hline Step 2: & $\begin{array}{l}\text { Calculate the two gray values } G_{\max 1} \text { and } G_{\max 2}, \text { which correspond to the two } \\
\text { maximum probabilities in the histogram. }\end{array}$ \\
\hline Step 3: & $\begin{array}{l}\text { Seek the gray value } G_{T} \subset\left[G_{\max 1}, G_{\max 2}\right] . G_{T} \text { corresponds to the minimum } \\
\text { probability in the range }\left[G_{\max 1}, G_{\max 2}\right], G_{\max 1}<G_{T}<G_{\max 2} .\end{array}$ \\
\hline Step 4: & $\begin{array}{l}\text { Segment the filtered image } I_{2}(x, y) \text { based on the threshold } G_{T} \text { and obtain the binary } \\
\text { image } I_{3}(x, y) .\end{array}$ \\
\hline
\end{tabular}

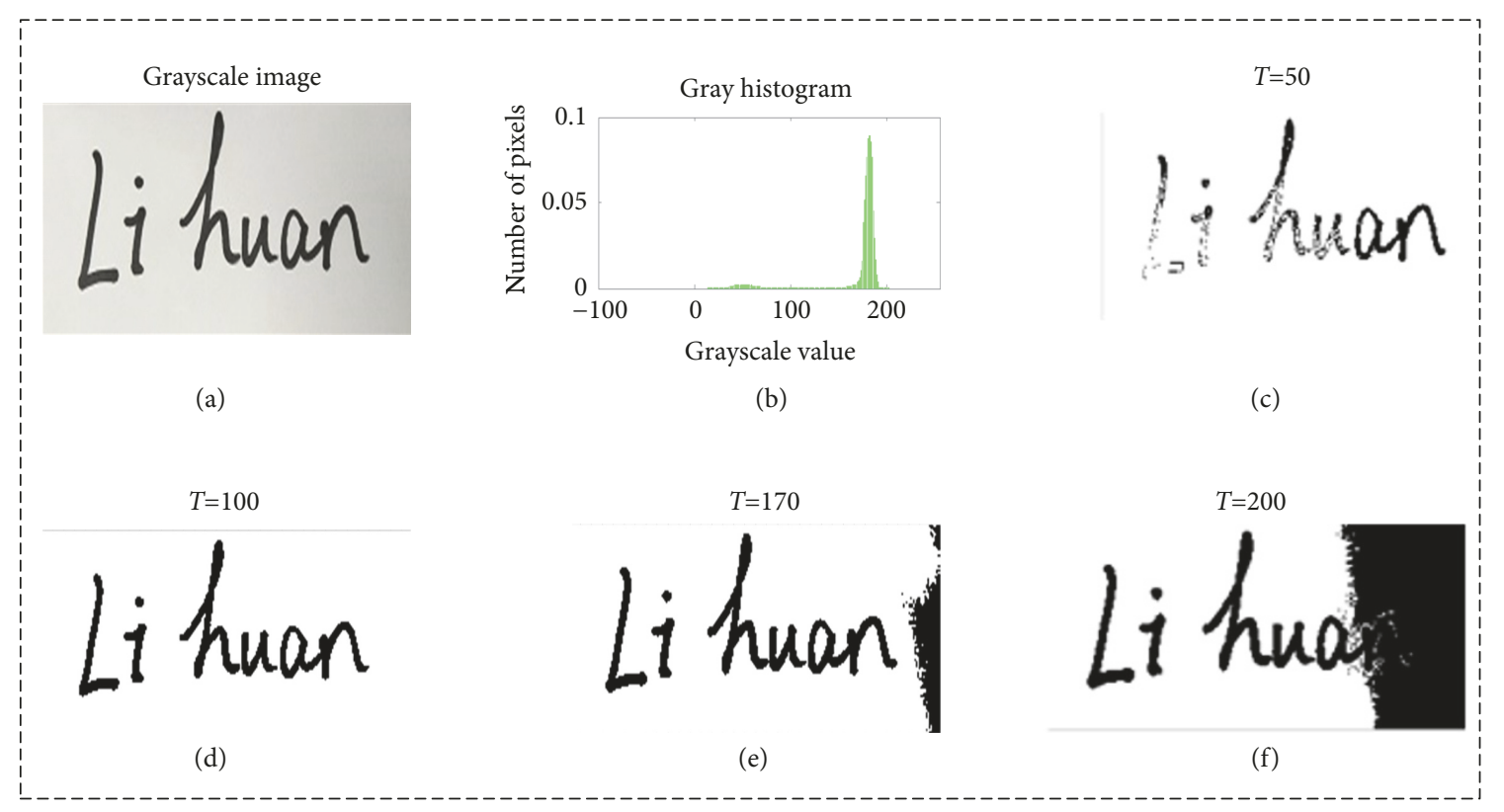

Figure 2: Gray signature image and the histogram program as well as the influence of a threshold on the segmentation result.

2.3. CNN Design For Decreasing Light Distortion. To ignore the light distortion and extract the frame construction and the special details of a signature, a CNN structure is designed, as shown in Figure 3. The CNN has the obvious advantages of local sensing, a hierarchical structure, and integration of the feature extraction and the classification. It is mainly used to verify the two-dimensional graph invariance if displacement, zoom, and other forms of distortion occur. This CNN structure is designed specifically for solving any light distortion problems.

First, the true $64 \times 128$ signature image is used as the input for $\mathrm{CNN}$ training. Second, we design the six convolution kernels $(9 \times 9)$ for the first stage of feature extraction of the true signature. The convolution layer $C_{1}$ is composed of six images $(56 \times 120)$. Third, the sampling kernel $S_{1}$ is selected with a size of $2 \times 2$ to obtain the pooling layer $\mathrm{P}_{1}$ which serves as the first part of the inputs to the SR classifier. Then, we design three convolution kernels $(5 \times 5)$ for feature extraction by the second stage.

The pooling layer $P_{1}$ is mapped to the convolution layer $C_{2}$ to extract the second level of features $P_{2}$, which serves as the second part of the inputs of the SR. In the pooling layers $P_{1}$ and $P_{2}$, the different images focus on different types of features. Some focus on the signature frame, some focus on the key points of a signature, and others focus on the changing areas of a signature. The designed $\mathrm{CNN}$ extracts relatively complete features from the original signature features. In this signature authentication system, the image features $P_{1}$ and $P_{2}$ work together and serve as the inputs of the SR. This CNN is applied to extract the frame construction and the special details of a signature, and eliminates any light distortion problems in a signature image.

2.4. Proposed SIA Algorithm To Increase The Recognition Accuracy. In this section, we design the SIA algorithm based on $\mathrm{CNN}$ and SR for signature authentication. The SIA algorithm is described in Table 3.

The SR uses the least number of suitable features for reconstruction of the most complete information. Therefore, the speed of the SR classifier is relatively high. The difficulty with the SR method is to determine the solution of the optimal objective function. Thus, we must solve the two 
TABLE 3: SIA algorithm for signature authentication.

\begin{tabular}{|c|c|}
\hline Input: & $\begin{array}{l}\text { Training samples of the true signature images, testing samples of the signature } \\
\text { images, and the given error parameter } \varepsilon \text {. }\end{array}$ \\
\hline Output: & The recognition result for the unknown signature. \\
\hline Step 1: & $\begin{array}{l}\text { Filter each sample by using the proposed golden G-L filtering algorithm and obtain } \\
\text { the sample set } Q_{1} \text {. }\end{array}$ \\
\hline Step 2: & $\begin{array}{l}\text { Segment each sample of } Q_{1} \text { using the developed MPT segmentation algorithm and } \\
\text { obtain the sample set } Q_{2} \text {. }\end{array}$ \\
\hline Step 3: & Design the CNN structure parameters as per Figure 3. \\
\hline Step 4: & $\begin{array}{l}\text { Build the CNN using the sample set } Q_{2} \text { and obtain the true signature feature images } \\
\text { of } P_{1} \text { and } P_{2} \text { from the designed CNN. }\end{array}$ \\
\hline Step 5: & $\begin{array}{l}\text { Calculate the over-complete dictionary } D \text { and the sparse coefficient nonzero } \\
\text { solutions } \mathrm{N} \text { by using } P_{1} \text { and } P_{2} \text {. }\end{array}$ \\
\hline Step 6: & Reconstruct the true signature template $S R=D * N$. \\
\hline Step 7: & $\begin{array}{l}\text { Reconstruct the unknown signature TSI using the same method as the true } \\
\text { signature template } S R \text {. }\end{array}$ \\
\hline Step 8: & $\begin{array}{l}\text { If }|T S I-S R| \leqq \varepsilon \text {, then the test signature is classified as true. Otherwise, the test } \\
\text { signature is classified as false. }\end{array}$ \\
\hline
\end{tabular}

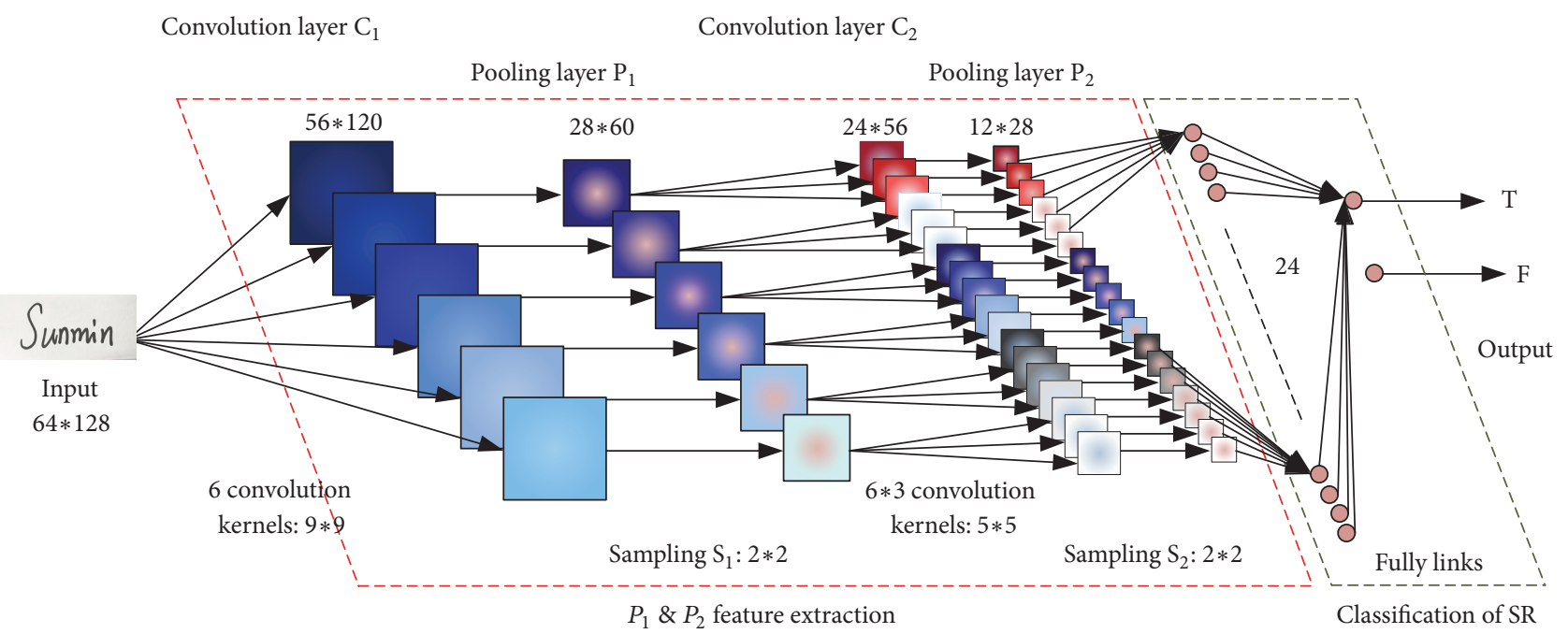

FIGURE 3: Designed CNN to solve the light distortion problem and extract the detailed frame features of a signature.

problems of obtaining the supercomplete dictionary and the nontrivial solution for sparse coefficients.

After the true signature image template SR is reconstructed, the test signature can be classified as true if the difference between the test signature image TSI and the reconstructed true signature image template SR is smaller than the error parameter $\varepsilon$. Otherwise, the test signature is classified as false.

\section{Experiments And Analysis}

In this section, we demonstrate three experiments of the G-L algorithm, the MPT algorithm, and the SIA algorithm followed by comparisons and discussion. The training and testing datasets of signatures are collected from 300 students. The students include 150 males and 150 females. The true signature number is 300 and the false signature number is also 300.

3.1. G-L Algorithm and MPT Algorithm Experiments. In Section 2.1, a new filtering algorithm, G-L, is developed, and the novel segmentation algorithm MPT is proposed in Section 2.2. Figure 4 shows the original signature image of the true signature and the fake signature. Figure 5 presents comparisons of the G-L filtering algorithm and the MPT segmentation algorithm to traditional methods.

The developed G-L filtering algorithm with added salt and pepper noise has the best filtering effect compared to traditional mean filtering, medium filtering, and Gaussian filtering. The developed G-L filtering algorithm decreases the influence of signature contour damage. 


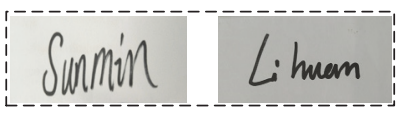

(a)

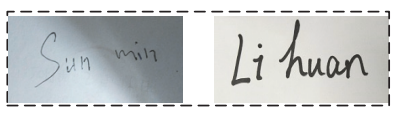

(b)

FIGURE 4: Original signature images: (a) true and (b) fake.

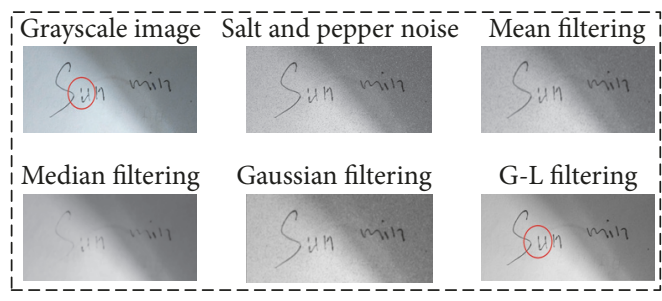

(a)

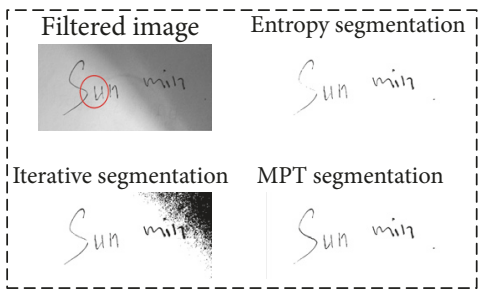

(b)

FIGURE 5: Experimental result comparisons of traditional methods and developed methods: (a) the filtering and (b) the segmentation.

The proposed MPT segmentation algorithm produces a better segmentation effect than the traditional methods of maximum entropy and iterative segmentation and has the minimum segmentation error.

3.2. SIA Algorithm Experiment. For the new SIA signature authentication algorithm, 90 true signature images and 72 false signature images for each user are considered. Twothirds of the samples of true and false images are used for CNN training, and the remaining one-third samples are used for $\mathrm{CNN}$ testing. Then, $P 1$ and $P 2$ obtained from the $\mathrm{CNN}$ are selected as the inputs of the SR. Figure 6 shows the signature features $P_{1}$ and $P_{2} . P_{1}$, and $P_{2}$ retain the frame features and the special detail features of the signature, ignoring any light distortion of the signature image.

For the SR of the SIA, the overcomplete dictionary $D$ and the sparse coefficients $N$ are essential. They are shown in Figures 7 and 8, respectively.

The process and result interface of the signature recognition system is shown in Figure 9. First, the CNN is trained using the training samples and the test samples. Then, the six signature frame features $P 1$ and the 18 detailed features $P 2$ are obtained, and the total 24 image features (which avoid the light distortion problem) serve as the inputs of the SR.

Finally, the dictionary $D$ and the sparse coefficients $N$ of the true signature are calculated, and the true signature template SR can be reconstructed. In Figure 9, a signature is input to the system to be judged as true or false by the proposed SIA algorithm. The right part shows the sparse coefficients of the 8 th chancel of the $S R$, the central part is the first 36 dictionaries of the SIZE, and the left pan shows the signature authentication result.

3.3. Comparisons and Discussion. Based on the theoretical analysis and the experiments, the comparison of traditional filtering methods and the developed golden G-L filtering algorithm is given in Table 4, the comparison of traditional segmentation methods and the developed MPT segmentation algorithm is given in Table 5, and the performance comparison of the traditional signature authentication methods and the proposed SIA algorithm is given in Table 6. The performance comparisons are shown in Table 7.

From Table 4, the proposed golden G-L filtering algorithm has the best signature contour damage mending and filtering result compared with the traditional methods of mean filtering, medium filtering, and Gaussian filtering. From Table 5, the developed MPT segmentation algorithm has the minimum segmentation error and produces the best signature segmentation compared with the traditional maximum entropy and iterative methods. From Table 6, the performance comparison results indicate that the proposed SIA algorithm has the highest signature authentication accuracy and acceptable time consumption performance compared with the traditional single $\mathrm{CNN}$ method and single SR method.

\section{Conclusions}

It is theoretically and experimentally verified that the proposed golden G-L algorithm has the best filtering result compared with the traditional methods of mean filtering, medium filtering, and Gaussian filtering in the case where the original signature contour is damaged. Meanwhile, the developed MPT algorithm has the best segmentation results with minimum error compared with the maximum entropy and iterative segmentation methods. In addition, the designed CNN can solve the light distortion problem for the feature extraction of the frame features and the detailed features of signature images. Finally, the proposed SIA algorithm achieves the highest average signature authentication accuracy of $97 \%$. In contrast, the average accuracies of the single CNN method and the single SR method are 95\% and 94\%, respectively. Consumption times are $0.8,1.0$, and $0.7 \mathrm{~s}$, respective to the proposed SIA, CNN, and SR. Future work will focus 
TABLE 4: Signature authentication comparisons.

\begin{tabular}{lcr}
\hline Filtering algorithm & Contour damage mending & Filtering result for signature \\
\hline Mean filtering & No & Better \\
\hline Medium filtering & No & Ordinary \\
\hline Gaussian filtering & No & Good \\
\hline G-L filtering & Yes & Best \\
\hline
\end{tabular}

TABLE 5: Signature authentication comparisons.

\begin{tabular}{lcc}
\hline Segmentation algorithm & Error rate & Segmentation effect \\
\hline Maximum entropy & Modest & Better \\
\hline Iterative method & Maximum & Ordinary \\
\hline MPT algorithm & Minimum & Best \\
\hline
\end{tabular}

TABLE 6: Comparison of signature authentication algorithms.

\begin{tabular}{lccc}
\hline Authentication algorithm & Accuracy & Time consumption \\
\hline CNN & Higher & Modest \\
\hline RS & Modest & Shortest \\
\hline SIA algorithm & Highest & Short & \\
\hline
\end{tabular}
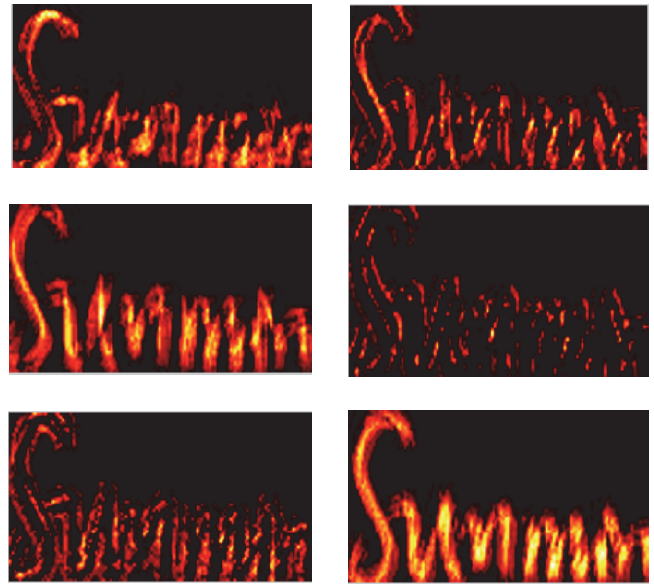

(a)
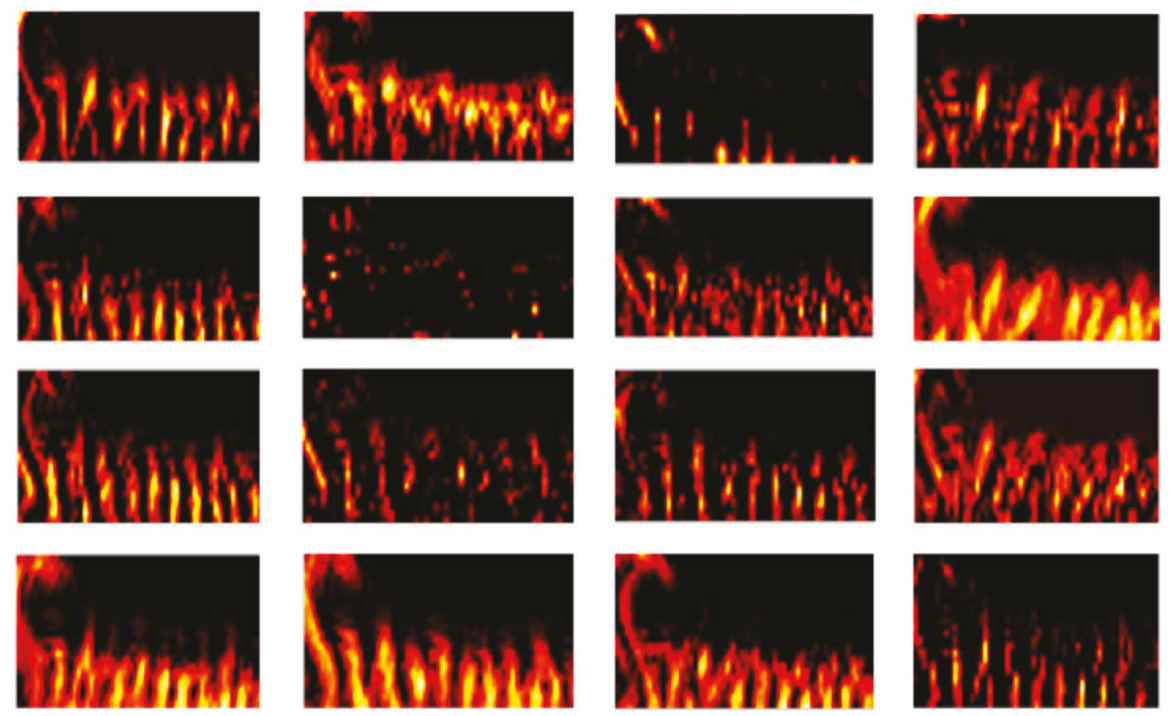

(b)

Figure 6: Image features obtained from the designed CNN: (a) $P_{1}$ and (b) $P_{2}$. 
TABLE 7: Performance comparisons.

\begin{tabular}{|c|c|c|c|c|c|c|}
\hline \multirow{2}{*}{ Test No. } & \multicolumn{2}{|c|}{ CNN } & \multicolumn{2}{|c|}{$\mathrm{RS}$} & \multicolumn{2}{|c|}{ SIA } \\
\hline & $\mathrm{A}(\%)$ & $\mathrm{T}(\mathrm{s})$ & $\mathrm{A}(\%)$ & $\mathrm{T}(\mathrm{s})$ & $\mathrm{A}(\%)$ & $\mathrm{T}(\mathrm{s})$ \\
\hline 1 & 96 & 0.8 & 93 & 0.6 & 99 & 0.6 \\
\hline 2 & 95 & 0.9 & 87 & 0.9 & 95 & 1.1 \\
\hline 3 & 94 & 1.0 & 95 & 0.5 & 99 & 0.7 \\
\hline 4 & 92 & 1.2 & 92 & 0.7 & 96 & 0.9 \\
\hline 5 & 93 & 1.1 & 91 & 0.8 & 96 & 0.7 \\
\hline Mean value & 95 & 1.0 & 94 & 0.7 & 97 & 0.8 \\
\hline
\end{tabular}

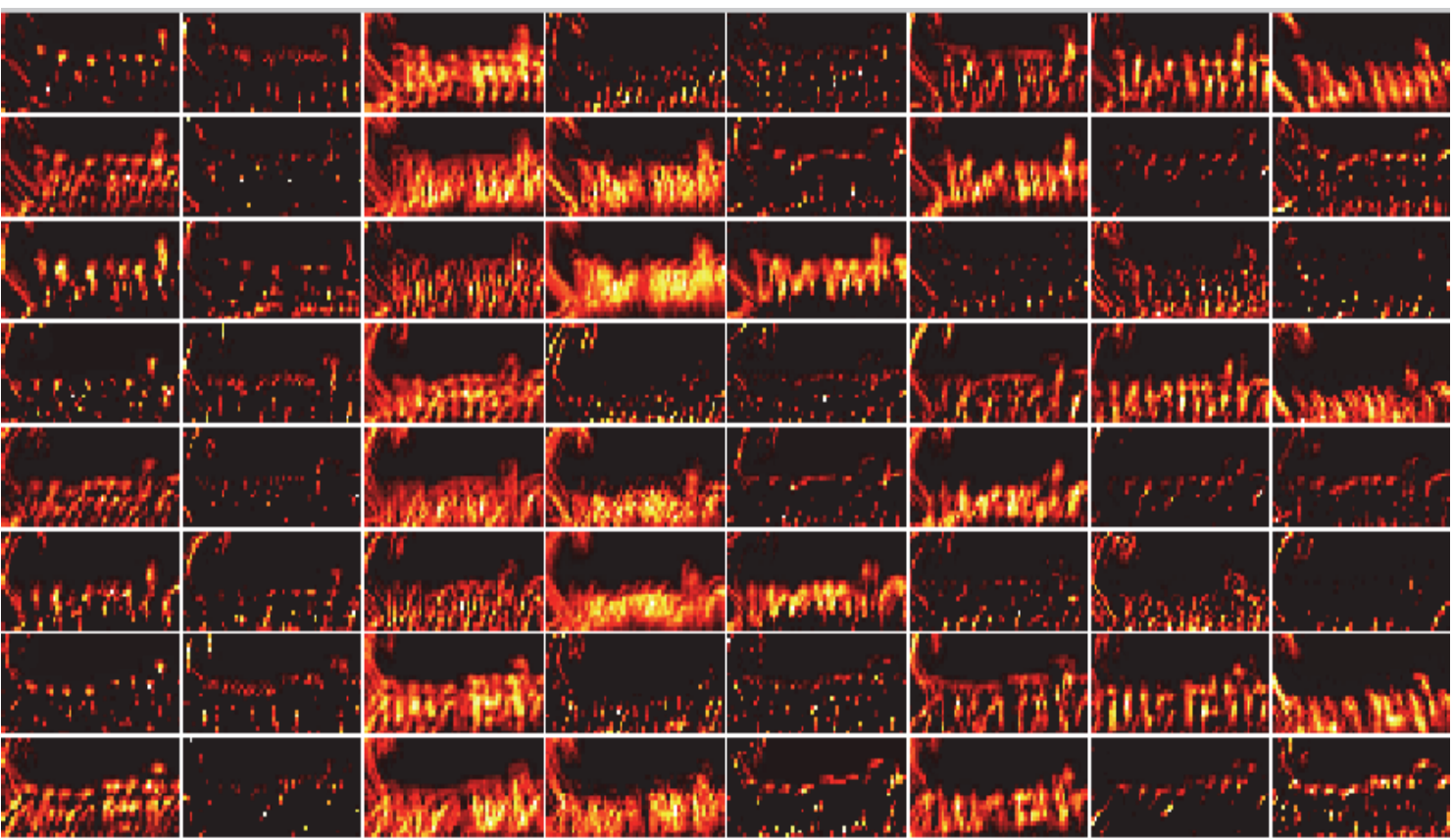

FIgURE 7: The first 64 overcomplete dictionaries $D$ of the SR.
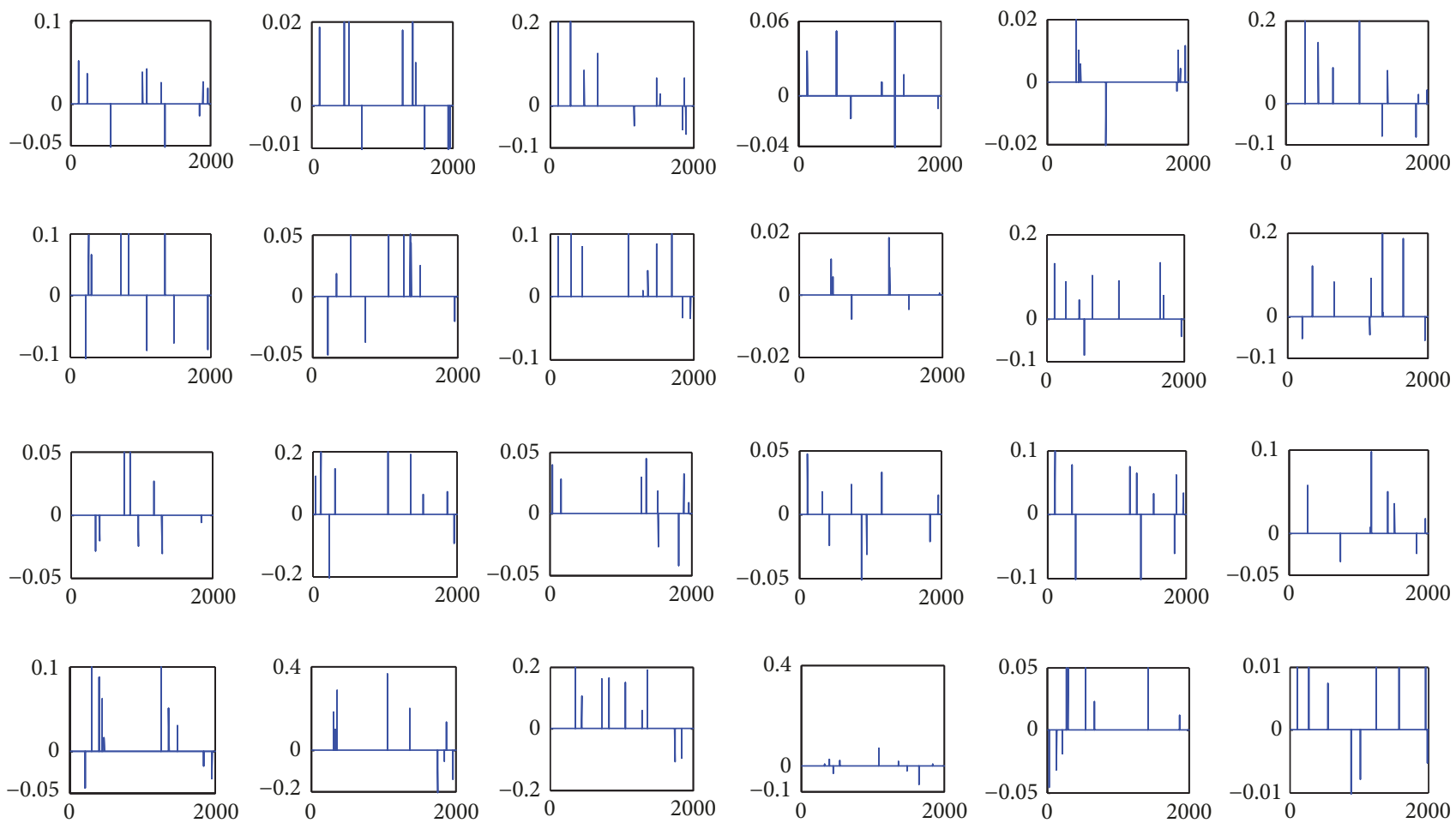

FIGURE 8: Sparse coefficients $N$ of the SR with 24 total channels. 


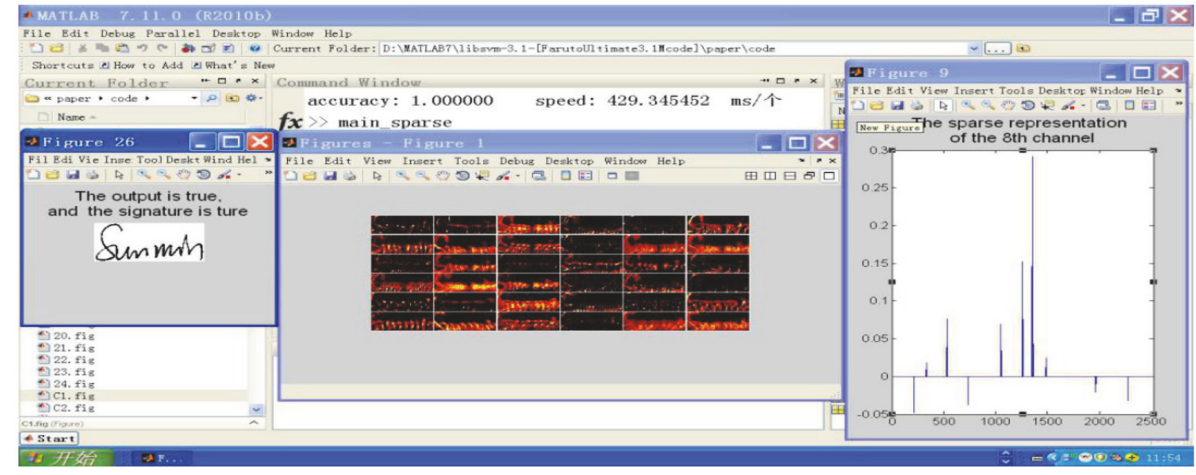

FIgURE 9: Signature authentication process and results.

on balancing and/or improving the performance between the signature authentication accuracy and the computation time of the proposed SIA algorithm.

\section{Conflicts of Interest}

The authors declare that they have no conflicts of interest.

\section{Acknowledgments}

This research was sponsored by the Natural Science Foundation of China (61300179), Key Scientific and Technological Project of Shaanxi Province (2016GY-040), and the Science Foundation of Xian University of Science and Technology (104-6319900001). We also thank Master students Huan Li and Min Sun for their support of data set collection.

\section{References}

[1] H. Lu, Y. Li, Y. Zhang, M. Chen, S. Serikawa, and H. Kim, "Underwater Optical Image Processing: a Comprehensive Review," Mobile Networks \& Applications, vol. 2017, pp. 1-12, 2017.

[2] A. Singh, P. Singh, A. Amini, and F. Marvasti, "Twin tree hierarchy: a regularized approach to construction of signature matrices for overloaded CDMA," Wireless Communications and Mobile Computing, vol. 16, no. 17, pp. 3070-3088, 2016.

[3] L. Yuan, Q. Ran, and T. Zhao, "Image authentication based on double-image encryption and partial phase decryption in nonseparable fractional Fourier domain," Optics \& Laser Technology, vol. 88, pp. 111-120, 2017.

[4] H. Wang, Y. Qin, Y. Huang, Z. Wang, and Y. Zhang, "Multipleimage encryption and authentication in interference-based scheme by aid of space multiplexing," Optics \& Laser Technology, vol. 95, pp. 63-71.

[5] K. S. Manjunatha, S. Manjunath, D. S. Guru, and M. T. Somashekara, "Online signature verification based on writer dependent features and classifiers," Pattern Recognition Letters, vol. 80, pp. 129-136, 2016.

[6] S. Serikawa and H. Lu, "Underwater image dehazing using joint trilateral filter," Computers and Electrical Engineering, vol. 40, no. 1, pp. 41-50, 2014.

[7] S. Lai, L. Jin, and W. Yang, "Toward high-performance online HCCR: A CNN approach with DropDistortion, path signature and spatial stochastic max-pooling," Pattern Recognition Letters, vol. 89, pp. 60-66, 2017.

[8] E. N. Zois, L. Alewijnse, and G. Economou, "Offline signature verification and quality characterization using poset-oriented grid features," Pattern Recognition, vol. 54, pp. 162-177, 2016.

[9] A. Sharma and S. Sundaram, "An enhanced contextual DTW based system for online signature verification using Vector Quantization," Pattern Recognition Letters, vol. 84, pp. 22-28, 2016.

[10] Y. Fang, W. Kang, Q. Wu, and L. Tang, "A novel video-based system for in-air signature verification," Computers \& Electrical Engineering, vol. 57, pp. 1-14, 2017.

[11] M. Wang, W.-Y. Chen, and X. D. Li, "Hand gesture recognition using valley circle feature and Hu's moments technique for robot movement control," Measurement, vol. 94, pp. 734-744, 2016.

[12] B. Kovari and H. Charaf, "A study on the consistency and significance of local features in off-line signature verification," Pattern Recognition Letters, vol. 34, no. 3, pp. 247-255, 2013.

[13] J. Canuto, B. Dorizzi, J. Montalvão, and L. Matos, "On the infinite clipping of handwritten signatures," Pattern Recognition Letters, vol. 79, pp. 38-43, 2016.

[14] R. Plamondon, C. O’Reilly, J. Galbally, A. Almaksour, and É. Anquetil, "Recent developments in the study of rapid human movements with the kinematic theory: Applications to handwriting and signature synthesis," Pattern Recognition Letters, vol. 35, no. 1, pp. 225-235, 2014.

[15] A. Soleimani, B. N. Araabi, and K. Fouladi, "Deep Multitask Metric Learning for Offline Signature Verification," Pattern Recognition Letters, vol. 80, pp. 84-90, 2016.

[16] A. B. De Oliveira, P. R. Da Silva, and D. A. C. Barone, "A novel 2D shape signature method based on complex network spectrum," Pattern Recognition Letters, vol. 63, article no. 6248, pp. 43-49, 2015.

[17] M. Wang, L. Guo, and W.-Y. Chen, "Blink detection using Adaboost and contour circle for fatigue recognition," Computers and Electrical Engineering, vol. 58, pp. 502-512, 2017.

[18] M. Wang, W. Qu, and W.-Y. Chen, "Hybrid sensing and encoding using pad phone for home robot control," Multimedia Tools and Applications, pp. 1-14, 2017.

[19] M. Wang, S. Zhang, Y. Lv, and H. Lu, "Anxiety Level Detection Using BCI of Miner's Smart Helmet," Mobile Networks and Applications, pp. 1-8, 2017. 
[20] Y. Liu, Z. Yang, and L. Yang, "Online Signature Verification Based on DCT and Sparse Representation," IEEE Transactions on Cybernetics, vol. 45, no. 11, pp. 2498-2511, 2015.

[21] P. Porwik, R. Doroz, and T. Orczyk, "Signatures verification based on PNN classifier optimised by PSO algorithm," Pattern Recognition, vol. 60, pp. 998-1014, 2016.

[22] H. Lu, Y. Li, T. Uemura et al., "FDCNet: filtering deep convolutional network for marine organism classification," Multimedia Tools and Applications, pp. 1-14, 2017.

[23] N. Kaothanthong, J. Chun, and T. Tokuyama, "Distance interior ratio: A new shape signature for $2 \mathrm{D}$ shape retrieval," Pattern Recognition Letters, vol. 78, pp. 14-21, 2016.

[24] X. Xia, Z. Chen, F. Luan, and X. Song, "Signature alignment based on GMM for on-line signature verification," Pattern Recognition, vol. 65, pp. 188-196, 2017.

[25] S. Ma, G. Chen, W. Wu, L. Song, X. Tian, and X. Wang, "Identifying effective initiators in OSNs: from the spectral radius perspective," Wireless Communications and Mobile Computing, vol. 16, no. 18, pp. 3340-3359, 2016.

[26] G. Goswami, P. Mittal, A. Majumdar, M. Vatsa, and R. Singh, "Group sparse representation based classification for multifeature multimodal biometrics," Information Fusion, vol. 32, no. 1, pp. 3-12, 2016.

[27] H. Liu, S. Li, and L. Fang, "Robust Object Tracking Based on Principal Component Analysis and Local Sparse Representation," IEEE Transactions on Instrumentation and Measurement, vol. 64, no. 11, pp. 2863-2875, 2015.

[28] B. Hou, B. Ren, G. Ju, H. Li, L. Jiao, and J. Zhao, "SAR image classification via hierarchical sparse representation and multisize patch features," IEEE Geoscience and Remote Sensing Letters, vol. 13, no. 1, pp. 33-37, 2016.

[29] Y. Shao, N. Sang, C. Gao, and L. Ma, "Probabilistic class structure regularized sparse representation graph for semisupervised hyperspectral image classification," Pattern Recognition, vol. 63, pp. 102-114, 2017.

[30] J. H. Cheon and M.-K. Lee, "Improved batch verification of signatures using generalized sparse exponents," Computer Standards \& Interfaces, vol. 40, pp. 42-52, 2015. 


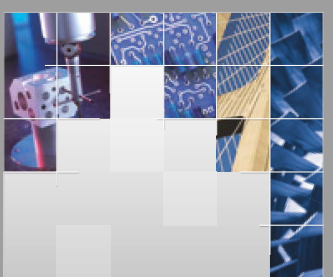

\section{Enfincering}
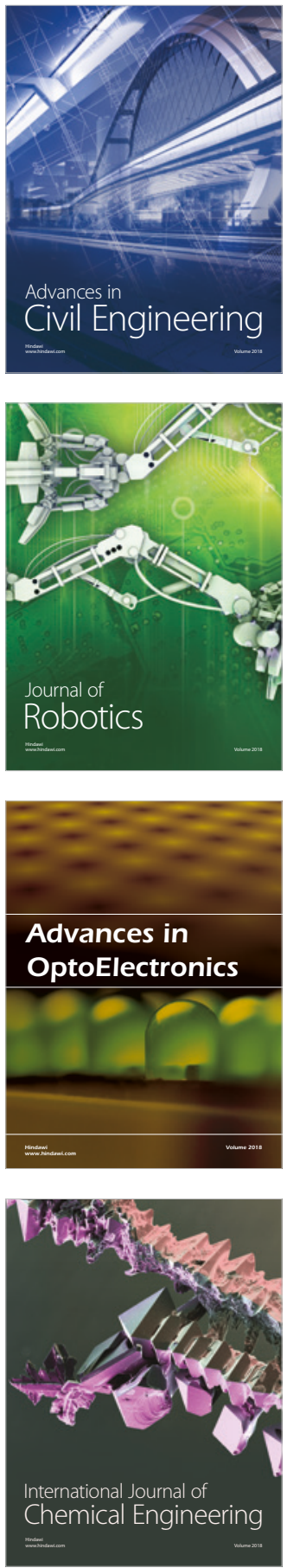

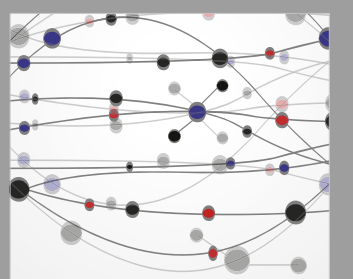

\section{Rotating \\ Machinery}

The Scientific World Journal

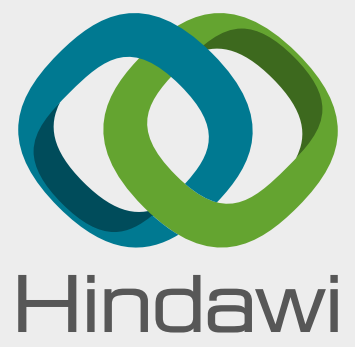

Submit your manuscripts at

www.hindawi.com
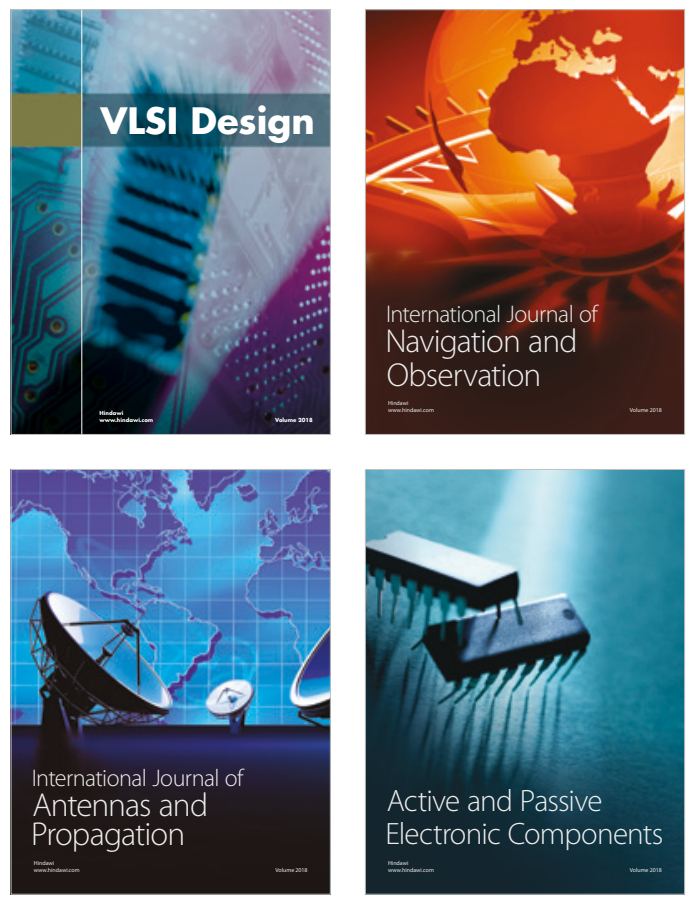
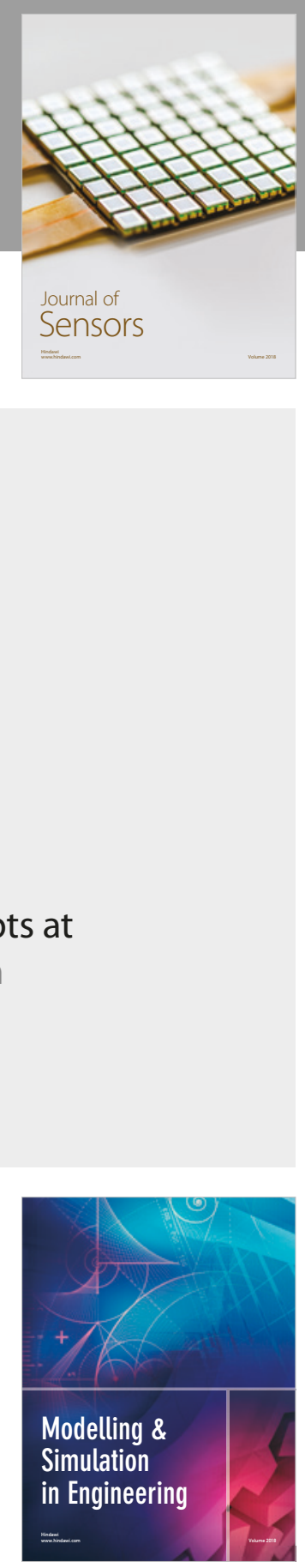

\section{Advances \\ Multimedia}
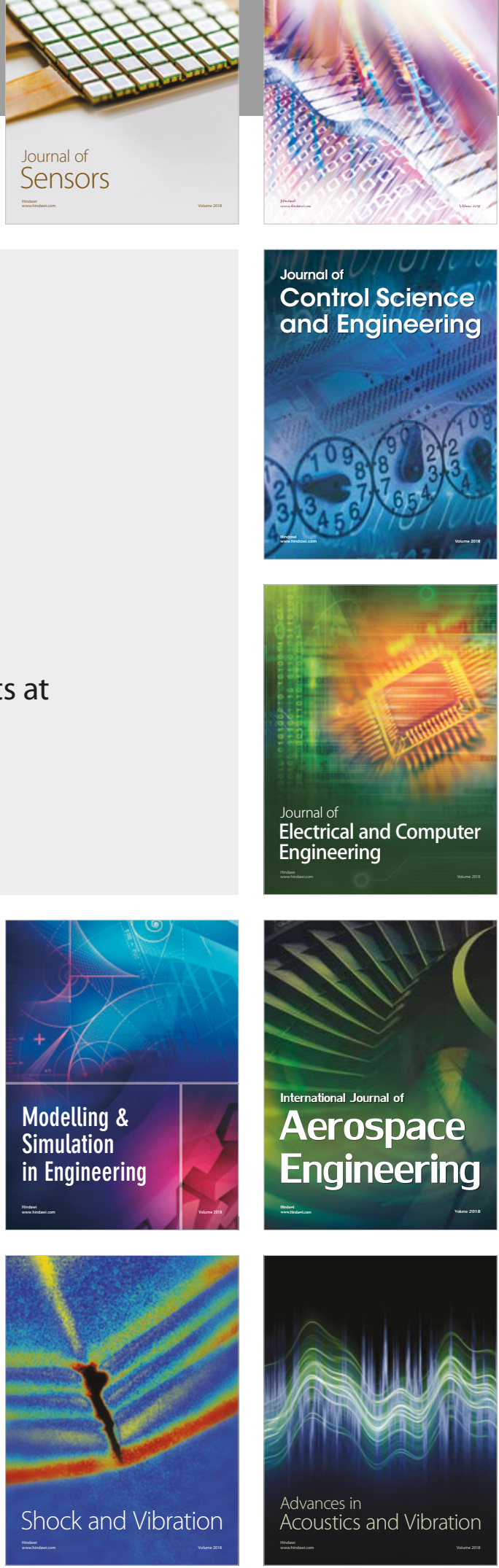